\title{
Differences in electromyographic activity of biceps brachii and brachioradialis while performing three variants of curl
}

\author{
Giuseppe Marcolin ${ }^{\text {Corresp.. }}{ }^{1}$, Fausto Antonio Panizzolo ${ }^{2}$, Nicola Petrone ${ }^{3}$, Tatiana Moro ${ }^{1}$, Davide Grigoletto ${ }^{1}$, \\ Davide Piccolo $^{4}$, Antonio Paoli ${ }^{1}$ \\ 1 Department of Biomedical Sciences, University of Padova, Padova, Italy \\ 2 John A. Paulson School of Engineering and Applied Sciences, Harvard University, Cambridge, United States \\ 3 Department of Industrial Engineering, University of Padova, Padova, Italy \\ 4 School of Sport Science, University of Padova, Padova, Italy \\ Corresponding Author: Giuseppe Marcolin \\ Email address: giuseppe.marcolin@unipd.it
}

Background. Dumbbell curl (DC) and barbell curl in its two variants, straight (BC) or undulated bar (EZ) are typical exercises to train the elbow flexors. The aim of the study was to verify if the execution of these three variants could induce a selective electromyographic (EMG) activity of the biceps brachii (BB) and brachioradialis (BR).

Methods. Twelve participants performed one set of ten repetitions at $65 \%$ of their 1-RM for each variant of curl. Pre-gelled electrodes were applied with an inter-electrode distance of $24 \mathrm{~mm}$ on BB and BR. An electrical goniometer was synchronously recorded with EMG signals to determine the concentric and eccentric phases of each variant of curl. Results. We detected higher activation profile of both $\mathrm{BB}(P<0.05)$ and $\mathrm{BR}(P<0.01)$ during the $\mathrm{EZ}$ compared to the DC. Higher levels of activation was found during the concentric phase for only the BR performed with an EZ compared to DC $(P<0.001)$ and performing BC compared to DC $(P<0.05)$. The eccentric phase showed a higher activation of the BB muscle in EZ compared to $\mathrm{DC}(P<0.01)$ and in $\mathrm{BC}$ compared to $\mathrm{DC}(P<0.05)$. BR muscle showed a higher activation performing EZ compared to $D C(P<0.01)$. Discussion. The $E Z$ variant may be preferred over the DC variant as it enhances BB and BR EMG activity during the whole range of motion and only in the eccentric phase. The small difference between $B C$ and $E Z$ variants of the $B B$ and BR EMG activity makes the choice between these two exercises a matter of subjective comfort. 
1 Differences in electromyographic activity of biceps brachii and brachioradialis while

\section{2 performing three variants of curl}

3

4 Giuseppe Marcolin ${ }^{1}$, Fausto Antonio Panizzolo², Nicola Petrone ${ }^{3}$, Tatiana Moro ${ }^{1}$, Davide

5 Grigoletto $^{1}$, Davide Piccolo ${ }^{4}$ and Antonio Paoli ${ }^{1}$

6

$7{ }^{1}$ Department of Biomedical Science, University of Padova, Via Marzolo 3, 35131 Padova, Italy

$8{ }^{2}$ John A. Paulson School of Engineering and Applied Sciences, Harvard University, Cambridge, 9 MA, USA

$10{ }^{3}$ Department of Industrial Engineering, University of Padova, Via Venezia 1, 35131 Padova, 11 Italy

$12{ }^{4}$ School of Sport Science, University of Padova, Via Marzolo 3, 35131 Padova, Italy

14 Corresponding author: Giuseppe Marcolin, PhD.

Department of Biomedical Science, University of Padova,

16 Via Marzolo 3, 35131 Padova, Italy

17 E-mail: giuseppe.marcolin@unipd.it 


\section{ABSTRACT}

Background. Dumbbell curl (DC) and barbell curl in its two variants, straight (BC) or undulated bar (EZ) are typical exercises to train the elbow flexors. The aim of the study was to verify if the execution of these three variants could induce a selective electromyographic (EMG) activity of the biceps brachii (BB) and brachioradialis (BR).

Methods. Twelve participants performed one set of ten repetitions at $65 \%$ of their 1-RM for each variant of curl. Pre-gelled electrodes were applied with an inter-electrode distance of $24 \mathrm{~mm}$ on $\mathrm{BB}$ and BR. An electrical goniometer was synchronously recorded with EMG signals to determine the concentric and eccentric phases of each variant of curl.

Results. We detected higher activation profile of both BB $(P<0.05)$ and $\mathrm{BR}(P<0.01)$ during the EZ compared to the DC. Higher levels of activation was found during the concentric phase for only the BR performed with an EZ compared to $\mathrm{DC}(P<0.001)$ and performing $\mathrm{BC}$ compared to DC $(P<0.05)$. The eccentric phase showed a higher activation of the BB muscle in EZ compared to $\mathrm{DC}(P<0.01)$ and in $\mathrm{BC}$ compared to $\mathrm{DC}(P<0.05)$. BR muscle showed a higher activation performing EZ compared to DC $(P<0.01)$.

Discussion. The EZ variant may be preferred over the $\mathrm{DC}$ variant as it enhances $\mathrm{BB}$ and $\mathrm{BR}$ EMG activity during the whole range of motion and only in the eccentric phase. The small difference between $\mathrm{BC}$ and $\mathrm{EZ}$ variants of the $\mathrm{BB}$ and $\mathrm{BR}$ EMG activity makes the choice between these two exercises a matter of subjective comfort. 


\section{INTRODUCTION}

55 Resistance training has fundamental importance in improving athletic performance as it allows the increase of muscular strength, power and speed (Kraemer \& Ratamess, 2004). In strength

57 athletes such as bodybuilders, resistance training plays a key role as it induces muscle hypertrophy. Therefore, to maximize muscle hypertrophy, bodybuilders construct training programs which involve exercises isolating specific muscles with different exercise variants or different ranges of movement, with the aim to increase muscle activity.

62 which target specific muscle groups. For instance, the effect of feet position on the electromyographic (EMG) activity of quadriceps muscles has been previously reported (Boyden,

64 Kingman, \& Dyson, 2000; Signorile, Kwiatkowski \& Caruso, 1995), as well as the effect of stance width on the activity of the gluteus maximus during a back squat (Paoli, Marcolin \& Petrone, 2009). In addition, EMG of the shoulder and trunk muscles during different variations

67 of the lat pull down exercise was investigated (Lusk, Hale \& Russell, 2005; Signorile, Zink, \&

68 Szwed, 2002), showing that maximal activation of the latissimus dorsi was obtained when

69 performing the exercise with an anterior wide grip. The effect of grip width and forearm 
70 pronation/supination on upper body muscles while performing the flat bench-press exercise was

71 also explored and it was found that small changes in muscle activity were associated with

72 changes in grip width (Lehman, 2005).

73 Previous studies have found that changes in the technical execution of an exercise could selectively influence muscle activity though there is very little information available focusing on exercises targeting the elbow flexors (biceps, brachialis and brachioradialis). Oliveira and Goncalves (Oliveira \& Gonçalves, 2009) found that different body posture influenced the demand of neuromuscular, cardiovascular and sensorial responses. Another study (Oliveira, \& al., 2009) reported that standing dumbbell curls and sitting dumbbell curls with the trunk inclined backwards were recommended for biceps force improvement. This was due to an overall higher neuromuscular effort in the whole range of motion while in the dumbbell preacher curls the activation of the biceps was maximal only for elbow angles close to full extension.

As reported in bodybuilder manuals (Hatfield, 1993) it is well accepted that two of the most employed dumbbell curls, the incline curls and the hummer curls, pre-stretch the biceps long head and enhance the involvement of the brachialis, respectively. On the other end, a very popular barbell curl named the Scott curls, unload the long head of the biceps placing greater overloading on the short head (Hatfield, 1993). Furthermore, performing barbell curls with a reverse handgrip resulted in an increase in brachioradialis activation (Hatfield, 1993). These exercises aim at inducing muscular hypertrophy of the elbow flexors, and can be performed using a straight bar, an undulated bar (named "EZ") or dumbbells.

To extend the findings of previous work and considering that there is no clear consensus

91 in the employment of the straight bar rather than the undulated bar or the dumbbells when the 92 aim is to increase the EMG activity of the biceps brachii and brachioradialis, the purpose of the 
93 present study was to investigate if the execution of barbell curls with straight bar (BC), barbell 94 curls with undulated bar (EZ) and alternate dumbbell curls (DC), affect the EMG activity of

95 elbow flexors. Furthermore, we hypothesized that EZ variant will induce a higher EMG activity 96 of the biceps brachii and brachioradialis due to the almost semiprone forearm position which 97 would increase the activation of the two muscles (Basmajian \& De Luca, 1978).

98

\section{MATERIALS AND METHODS}

Twelve male participants (age $25 \pm 5$ years, body mass $77 \pm 9 \mathrm{~kg}$, height $183 \pm 6 \mathrm{~cm}$ ) with at least three years resistance training experience (three resistance training sessions per week) were recruited in the present study. All participants had to habitually train with both dumbbell and barbell curls and were right hand dominant. At the time of the study the participants did not present any pathology of the shoulders, elbows and wrists and they were free from neuromuscular diseases. A detailed description of the experimental procedures was given to each participant and an informed consent was obtained. The experimental protocol adhered to the principles of the 1975 Helsinki Declaration and was approved by the ethical committee of the Department of Biomedical Sciences, University of Padova (HEC-DSB12/16).

Muscle activity was recorded by means of a PDA Pocket EMG (BTS BioengineeringItaly). Device resolution was 16 bit, weight $300 \mathrm{~g}$ and dimensions $145 \times 95 \times 20 \mathrm{~mm}$. The sampling frequency was set to $1 \mathrm{kHz}$ to avoid aliasing phenomena. Muscles analyzed were the biceps brachii $(\mathrm{BB})$ and the brachioradialis $(\mathrm{BR})$ of the right limb of each participant. $\mathrm{Ag} / \mathrm{AgCl}$ pregelled electrodes were applied with an inter-electrode distance of $24 \mathrm{~mm}$. Skin preparation, sensor location and orientation on the muscles were in accordance with Hermens and colleagues (Hermens et al. 2000). In order to determine the concentric and the eccentric phases of the 
116 exercise, an electrical goniometer (Biometrics LTD, UK) was placed on the right elbow of each

117 participant and recorded at $1 \mathrm{kHz}$ (synchronously with EMG signals).

118 A standing posture was maintained during the performance of the three exercises: BC,

119 EZ, DC. The one repetition maximum (1-RM) was determined for each of the three exercises by

120 means of a submaximal estimation method during three separate days as detailed in Brzycki

121 (Brzycki, 1993). The experimental data collection were carried out in the biomechanical

122 laboratory and performed on the same day for all participants. Each participant performed a

123 standardized warm-up consisting of 12 repetitions of alternate dumbbell curls at $40 \%$ of his 1 -

124 RM. The participants were then asked to execute 1 set of 10 repetitions for each of the three

125 variants in a randomized order. A metronome was employed to impose the same time of

126 execution among sets and among participants. The metronome was set so that each repetition

127 duration was 3 seconds. The load employed corresponded to $65 \%$ of the 1 -RM. The rest between

128 sets was fixed at 4 minutes to allow an adequate recovery. During the execution of the repetitions

129 participants had to assume a standing posture and to follow the rhythm imposed by the

130 metronome as much as possible. Trunk movements together with knee flexions were visually

131 monitored by the researchers to avoid possible cheating. For better clarity a scheme of the

132 experimental protocol is reported in figure 1.

133 To obtain a linear envelop, EMG interference signals were first rectified around their

134 mean value, then integrated with a moving window of 200 milliseconds, and finally smoothed

135 with a $4^{\text {th }}$ order Butterworth low pass filter set at $5 \mathrm{~Hz}$. The concentric phase was defined from

136 elbow maximal extension to maximal flexion, the eccentric phase was defined from elbow

137 maximal flexion up to maximal extension, obtained by means of the electrical goniometer. The

138 first and last repetitions (out of 10) were not selected for analysis due to the inconsistencies in 
139 technique (Paoli, Marcolin, \& Petrone, 2009, 2010). For each participant and each experimental

140 condition, the mean EMG activity was computed for each repetition (relative to the concentric,

141 eccentric phase and whole movement) and an average of the eight repetitions was the calculated

142 and reported for each of the three exercises, both for BB and BR.

143 Friedman non-parametric test for repeated measurements was used to compare the three

144 exercises. Significant level was set at $P<0.05$. If a statistically significant difference was found,

145 Dunn's multiple comparison test was employed. Data analysis was performed by means of the

146 software package GraphPad Prism version 4.00 for Windows (GraphPad Software, San Diego

147 California USA). Statistical effect size was calculated with the G*Power 3.1.5 software (Faul, et

148 al., 2007).

\section{RESULTS}

151 The time of execution considering all the repetitions of the three variants was $3.16 \pm 0.67 \mathrm{~s}$. In 152 particular the concentric movement duration was $1.44 \pm 0.26 \mathrm{~s}$ while the eccentric movement 153 was $1.72 \pm 0.47 \mathrm{~s}$. The range of motion (ROM) mean of the right elbow (figure 2 ) recorded in the 154 three variants of $\operatorname{curl}\left(\mathrm{BC}=117.3^{\circ} \pm 10.9^{\circ} ; \mathrm{EZ}=119.9^{\circ} \pm 13.7^{\circ} ; \mathrm{DC}=123.1^{\circ} \pm 12.3^{\circ}\right)$ was found to be statistically different $(P=0.0087)$. However, post hoc test showed only a greater ROM in BC with respect to $\mathrm{DC}(\mathrm{P}<0.05, E S=0.46)$. In figure 3 we reported an example of representative EMG data (Biceps activity of 1 subject in the three variants of curl). Considering the whole range of motion (figure 4-A) significant differences were observed in the EMG activity of the BB $(P=0.0204)$ and $\mathrm{BR}(P=0.0023)$. Post hoc test showed a higher activation during the EZ variant with respect to the $\mathrm{DC}$ both for $\mathrm{BB}(P<0.05, E S=0.30)$ and $\mathrm{BR}(P<0.01, E S=0.51)$. No differences were detected between $\mathrm{EZ}$ and $\mathrm{BC}$ for both muscles investigated. 
The concentric movement (figure 4-B) showed statistically significant differences for the $\mathrm{BR}(P=0.0009)$ but not for the BB. Post hoc test showed a higher activation of the BR during EZ

164

165

166

167

168

169

170

171

172

173

174

175

176

177

178

179

180

181

182

183

184

variant with respect to $\mathrm{DC}(P<0.001, E S=0.48)$ and of the $\mathrm{BC}$ with respect to $\mathrm{DC}(P<0.05$, $E S=0.36)$.

The eccentric movement (figure 4-C) showed a difference in the EMG activity for the BB $(P=0.0014)$. Specifically, muscle activity was higher in the EZ variant compared to the DC variant $(P<0.01, E S=0.45)$ as well as in the $\mathrm{BC}$ variant compared to the $\mathrm{DC}(P<0.05, E S=0.28)$.

BR muscle showed statistically significant differences during the eccentric phase $(P=0.0038)$ with a higher activation during the EZ variant in comparison to the DC variant $(P<0.01$, $E S=0.66)$.

\section{DISCUSSION}

The enhancement of the activity of specific muscles with different exercise variants and different ranges of motion have been extensively examined for both the lower limb muscles (Boyden et al., 2000; Paoli, Marcolin \& Petrone, 2009; Signorile, Kwiatkowski \& Caruso, 1995) and trunk muscles (Lehman, 2005; Lusk, Hale \& Russell, 2005; Marcolin et al., 2015; Paoli, Marcolin \& Petrone, 2010; Signorile et al., 2002). Nevertheless, few studies have investigated the EMG activity of the arm and forearm muscles while performing resistance exercises (Oliveira \& Gonçalves, 2009; Oliveira et al., 2009). To the best of our knowledge, this was the first study to assess the influence of different variants of the curl exercise on the level of activation of the BB and BR muscle.

In the present work, the EZ variant exhibited the highest level of EMG activity for both $\mathrm{BB}$ and BR. However, significant differences were observed only in comparison with the DC 
185 variant. The concentric phase analysis of the BB showed that the EZ variant induced the highest

186 level of EMG activity ( $+7 \%$ with respect to $\mathrm{BC}$ and $+11 \%$ with respect to $\mathrm{DC}$ ) although there

187 were no statistically significant differences. Conversely, the variation of curls on BR muscle

188 activity during the concentric phase resulted to be statistically significant when comparing the

189 EZ to the DC as well as the BC to the DC.

190 The eccentric phase showed lower EMG activity with respect to the concentric phase for 191 both muscles investigated. Specifically, EZ and BC variants elicited a statistically significant 192 higher EMG activity of the BB muscle with respect to the DC variant. BR muscle activity was 193 found to be significantly higher only in the EZ variant compared to the DC.

As the comparison was made between two bilateral movements (BC and $\mathrm{EZ})$ and one unilateral movement (DC), our results could have been affected by the phenomenon of the bilateral deficit (Howard \& Enoka, 1991) which showed a higher EMG activity in the unilateral movement (DC). Nevertheless it seems that this was not the case in our study. Most it is possible that our findings were not affected by the bilateral facilitation (Botton et al., 2016) as all participants were equally familiar with both the $\mathrm{BC}$ or $\mathrm{EZ}$ and the $\mathrm{DC}$ variants.

Therefore, the anatomical aspects of these two muscles have to be taken into account when interpreting the results. Biceps brachii is a robust forearm supinator and an elbow flexor while brachioradialis, the most superficial muscle of the forearm considering the radial part, acts as an elbow flexor (Williams et al., 1989). Wire EMG analysis suggested that these two muscles, together with the brachialis, differ in their flexor activity depending on the three positions of the forearm: prone, semiprone and supine (Basmajian \& De Luca, 1978). During the execution of the three exercise variants in the present study, the forearms assumed different positions through the dynamic elbow flexion movement. In the $\mathrm{BC}$, both forearms are supined throughout the 
208 performance of the exercise while in the EZ barbell curl they assume an intermediate position

209 very close to a semiproned position. In the DC, the forearm is semiproned at the initial phase of

210 the repetition, after which it assumes a supine position at approximately $90^{\circ}$ of elbow flexion

211 until the end of the concentric phase. The almost semiproned forearm position during the EZ

212 barbell curl could explain the higher muscle activity in this variant. Accordingly to Basmajan et

213 al. (Basmajian \& De Luca, 1978), they reported that the biceps brachii, brachialis and

214 brachioradialis act maximally when the weight is lifted by means of flexing the elbow

215 throughout a semiproned forearm position.

216 Although significant differences were found between variants, some limitations of the

217 present study need to be acknowledged. First, even if widely employed in kinesiologic and sport

218 applications it has been demonstrated that bipolar surface EMG signals can be influenced by the

219 thickness of the subcutaneous tissue layers, electrode size and shape, spatial filter transfer

220 function, and interelectrode distance (Farina, Cescon \& Merletti, 2001). Moreover the consensus

221 on the electrode placement is still debated (Mesin, Merletti \& Rainoldi, 2008). Second, the

222 present work did not assess EMG activation of the brachialis muscle. Even if it is still unclear

223 whether its activity can be accurately assessed with surface electrodes at high levels of muscular

224 contraction (Staudenmann \& Taube, 2015), its exclusion in the analysis of the three variants of

225 curl has to be acknowledged as a limitation to the present study since this muscle is one of the

226 main contributor during elbow flexion. Third, a different ROM was reported in the three

227 exercises variants. Nevertheless, the presence of little differences in term of ROM

228 (approximately less than $6^{\circ}$ ) was specific to the technical execution of each variant of curl and

229 thus standardizing its value could have prevented the participants optimally performing the 230 exercises. 


\section{CONCLUSION}

234 We can conclude that the EZ barbell curl was the most effective variant considering the overall EMG activity of the BB and BR. On the other hand, DC variant was found to be less effective, while $\mathrm{BC}$ variant could be placed in an intermediate position but with an activation closer to the EZ than to the DC variant for the two muscles investigated.

Our findings suggested that EZ barbell curl may be preferred to DC considering the whole phase of the repetition and the eccentric phase both for BB and BR. The small difference between BC and $\mathrm{EZ}$ variants with regards to the EMG activity of the $\mathrm{BB}$ and $\mathrm{BR}$, makes the choice between these two variants purely a matter of subjective comfort related to the handgrip position.

\section{ACKNOWLEDGEMENTS}

246 The authors would like to thank all the participants that took part in the study and Dr. Luqman 247 Aziz for reviewing the English style.

\section{REFERENCES}

Basmajian, J.V., De Luca CJ. Muscles Alive: Their Functions Revealed by Electromyography. Williams \& Wilkins, 1978.

Botton E, Radaelli R, Wilhelm EN, Rech A, Brown LE, Pinto RS. Neuromuscolar adaptations to 
254

255

256

257

258

259

260

261

262

263

264

265

266

267

268

269

270

271

272

273

274

275

276

unilateral vs. bilateral strength training in women. J Strength Cond Res 2016; 30(7):1924 1932.

Boyden G, Kingman J, Dyson R. A comparison of quadriceps electromyographic activity with the position of the foot during the parallel squat. J Strength Cond Res 2000; 14(4):379382.

Brzycki M. Strength testing-predicting a one-rep max from reps-to-fatigue. $J$ Phys Educ Recreat Danc 1993; 64(1):88-90.

Farina D, Cescon C, Merletti R. Influence of anatomical, physical, and detection-system parameters on surface EMG. Biol Cybern 2002; 86(6): 445-456.

Faul F, Erdfelder E, Lang A-G, Buchner A. G*Power 3: a flexible statistical power analysis program for the social, behavioral, and biomedical sciences. Behav Res Methods 2007; 39(2):175-191.

Hatfield F. Hardcore bodybuilding: a scientific approach. Chicago, Illinois, Contemporary Books, Inc, 1993.

Hermens HJ, Freriks B, Disselhorst-Klug C, Rau G. Development of recommendations for SEMG sensors and sensor placement procedures. J Electromyogr Kinesiol 2000; $10(5): 361-374$.

Howard JD, Enoka RM. Maximum bilateral contractions are modified by neurally mediated interlimb effects. J Appl Physiol 1991;70(1):306-16.

Kraemer WJ, Ratamess NA. Fundamentals of resistance training: progression and exercise prescription. Med Sci Sports Exerc 2004; 36(4):674-688.

Lehman GJ. The influence of grip width and forearm pronation/supination on upper-body myoelectric activity during the flat bench press. Strength Cond 2005; 19(3):587-591. 
277 Lusk, S.J., Hale, B.D., Russell DM. Grip width and forearm orientation effects on muscle

278 activity during the lat pull-down. J Strength Cond Res 2005; 24(7):1895-1900.

279 Marcolin G, Petrone N, Moro T, Battaglia G, Bianco A, Paoli A. Selective activation of

280 shoulder, trunk, and arm muscles: A comparative analysis of different push-up variants. $J$

281

282 Athl Train 2015; 50(11):1126-1132.

Mesin L, Merletti R, Rainoldi A. Surface EMG: The issue of electrode location. J Electromyogr Kinesiol 2009; 19(5):719-726.

Oliveira LF, Matta TT, Alves DS, Garcia MAC, Vieira TMM. Effect of the shoulder position on the biceps brachii EMG in different dumbbell curls. J Sport Sci Med 2009; 8(1):24-29.

Oliveira, A.S., Gonçalves M. Positioning during resistance elbow flexor exercise affects electromyographic activity, heart rate, and perceived exertion. J Strength Cond Res 2009;

Paoli, A., Marcolin, G., Petrone N. Influence of different ranges of motion on selective 23(3):854-862.

292

293

294

295

296

297

298

299 recruitment of shoulder muscles in the sitting military press: an electromyographic study. J Strength Cond Res 2010; 24(6):1578-1583.

Paoli, A., Marcolin, G., Petrone N. The effect of stance width on the electromyographical activity of eight superficial thigh muscles during back squat with different bar loads. $J$ Strength Cond Res 2009; 23(1):246-250.

Signorile JF, Zink AJ, Szwed SP. A comparative electromyographical investigation of muscle utilization patterns using various hand positions during the lat pull-down. J Strength Cond Res 2002; 16(4):539.

Signorile, J.F., Kwiatkowski, K., Caruso, J.F. RB. Effect of foot position on the electromyographycal activity of the superficial quadriceps muscles during the parallel 

squat and knee extension. J Strength Cond Res 1995; 9(3):182-187.

301 Staudenmann D, Taube W. Brachialis muscle activity can be assessed with surface electromyography. J Electromyogr Kinesiol 2015; 25(2):199-204.

303

304

305

306

307

308

309

310

311

312

313

314

315

316

317

318

319

320

321

322 323
Williams, P.L., Warwick, R., Dyson, M., Bannister LH. Gray’s anatomy. Edinburgh, Churchill livingstone, 1989. 
324 Figure 2 Mean values of the range of motion (ROM) recorded in the three variants of curl (* $325 \mathrm{p}<0.05)$.

326

327 Figure 3 Representative biceps brachii EMG data of 2 repetitions of the three variants of curl. 328 From top to bottom: (A) BB, (B) EZ and (C) DC.

329

330

Figure 4 EMG activity of the biceps brachii and brachioradialis during (A-B) the whole range of 331 motion, (C-D) the concentric phase, (E-F) the eccentric phase. $(* \mathrm{p}<0.05, * * \mathrm{p}<0.01$, *** $332 \mathrm{p}<0.001)$. 
Figure 1

Experimental protocol.

Graphical representation of the experimental protocol.

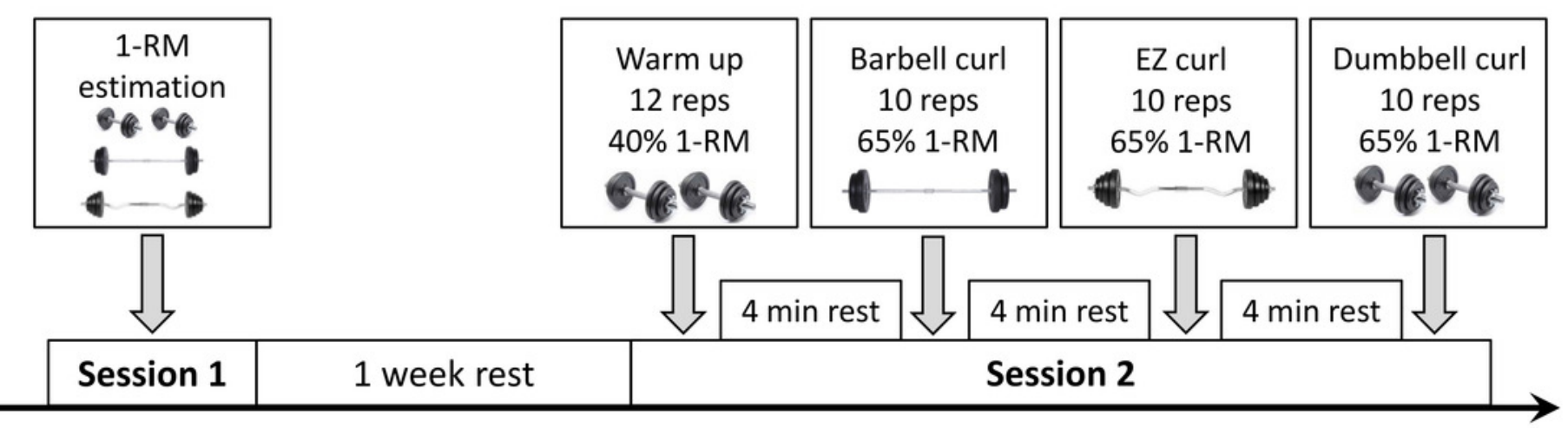


Figure 2

Range of motion.

Mean values of the range of motion (ROM) recorded in the three variants of curl $(* p<0.05)$.

\section{Elbow ROM}

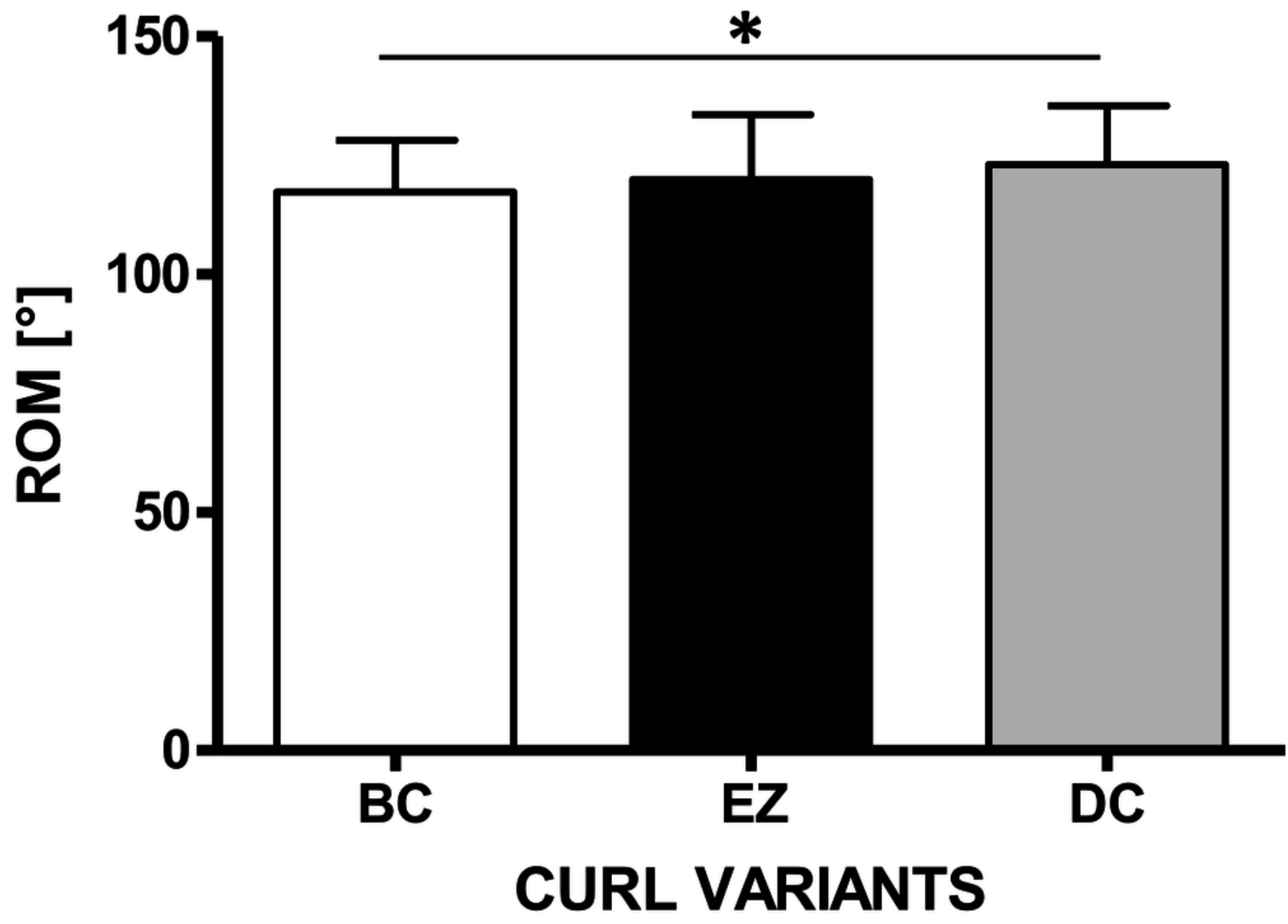


Figure 3

Representative EMG data.

Representative biceps brachii EMG data of 2 repetitions of the three variants of curl. From top to bottom: (A) BB, (B) EZ and (C) DC. 

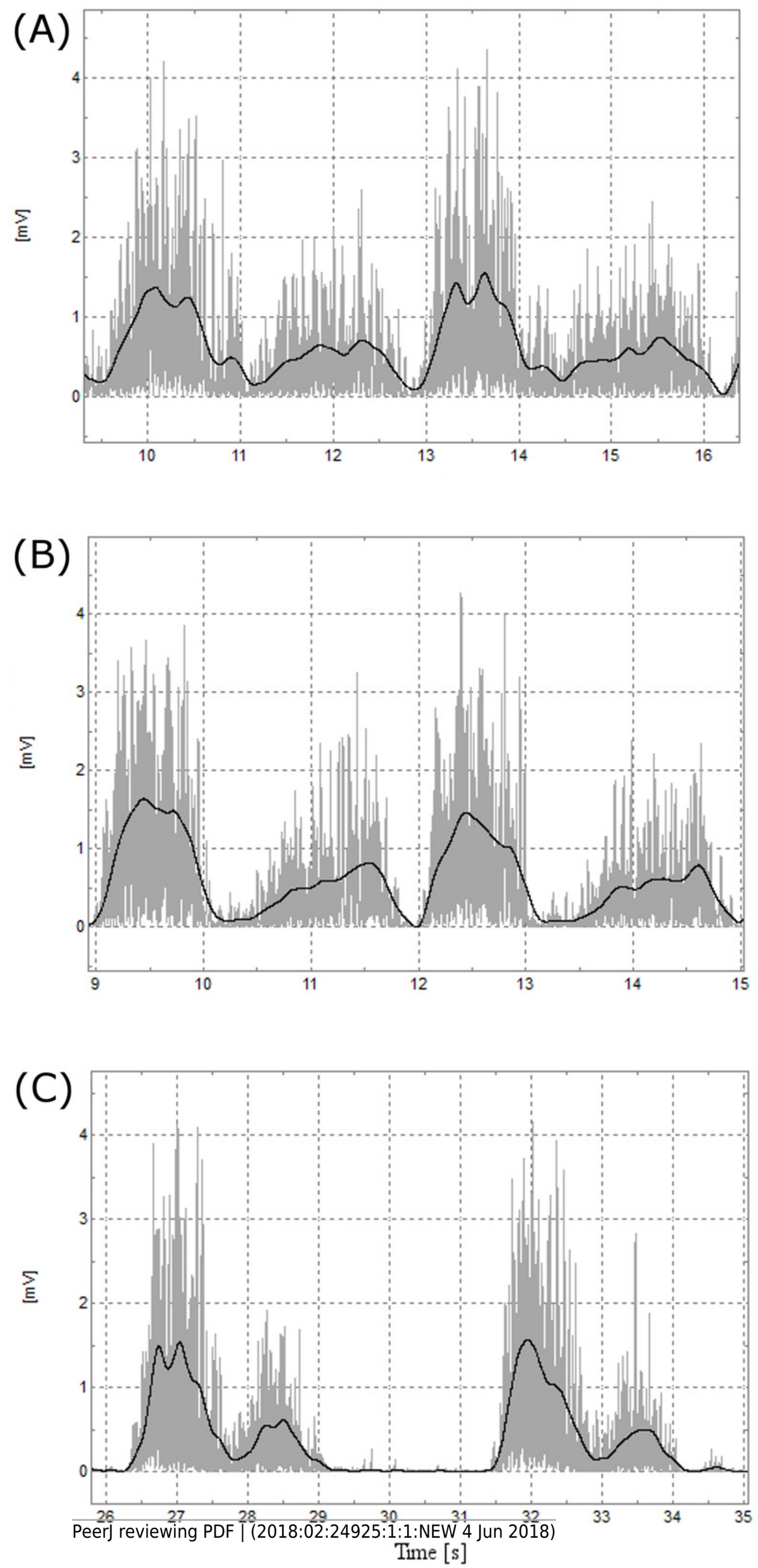
Figure 4

EMG activity.

EMG activity of the biceps brachii and brachioradialis during (A-B) the whole range of motion, (C-D) the concentric phase, $(E-F)$ the eccentric phase (C). $(* p<0.05, * * p<0.01)$. 
(A)

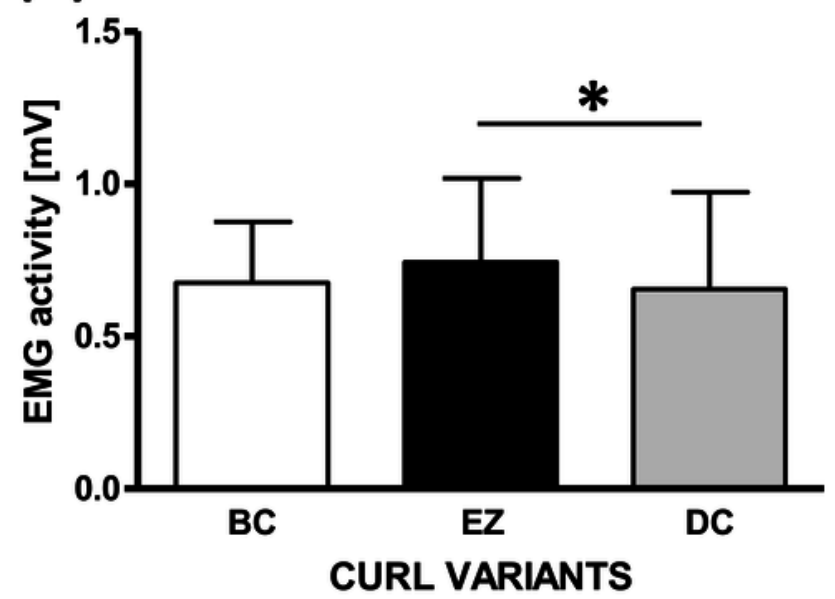

(C) biceps brachii

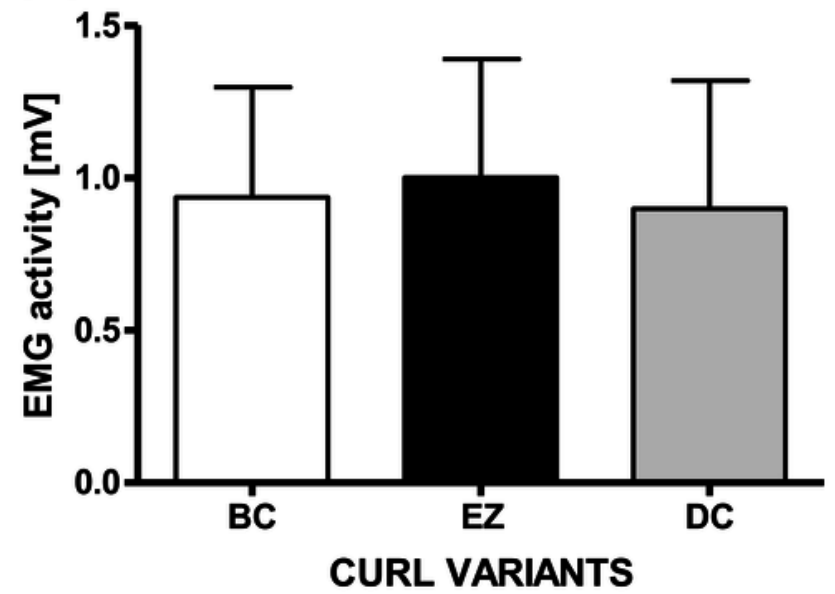

(E)

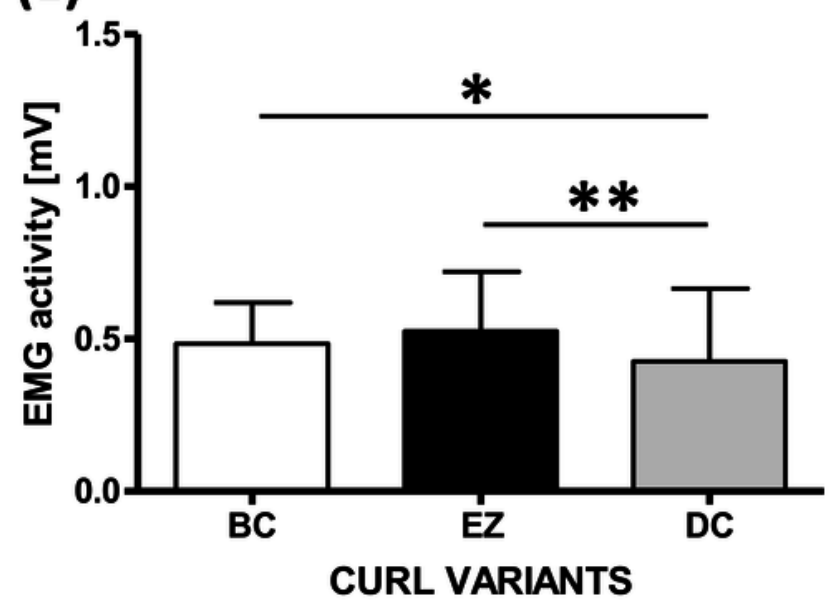

(B) brachioradialis

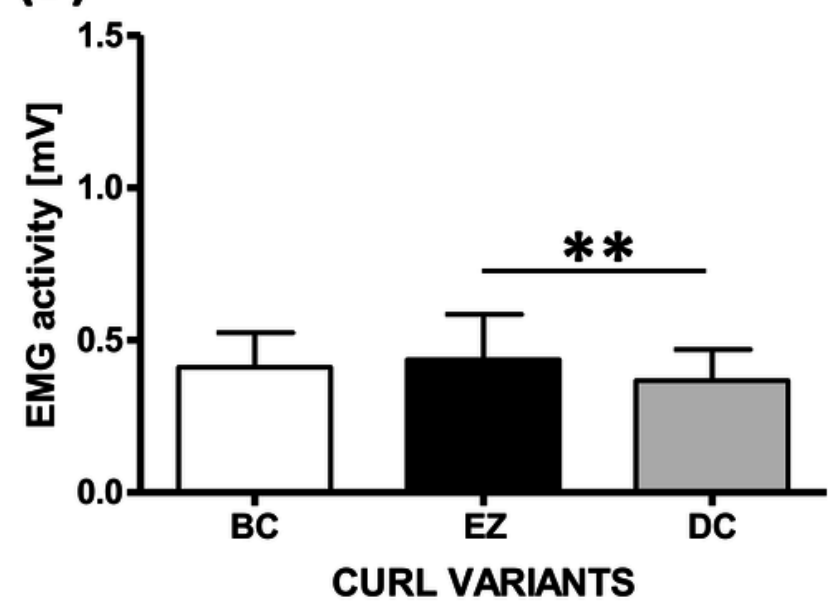

(D) brachioradialis

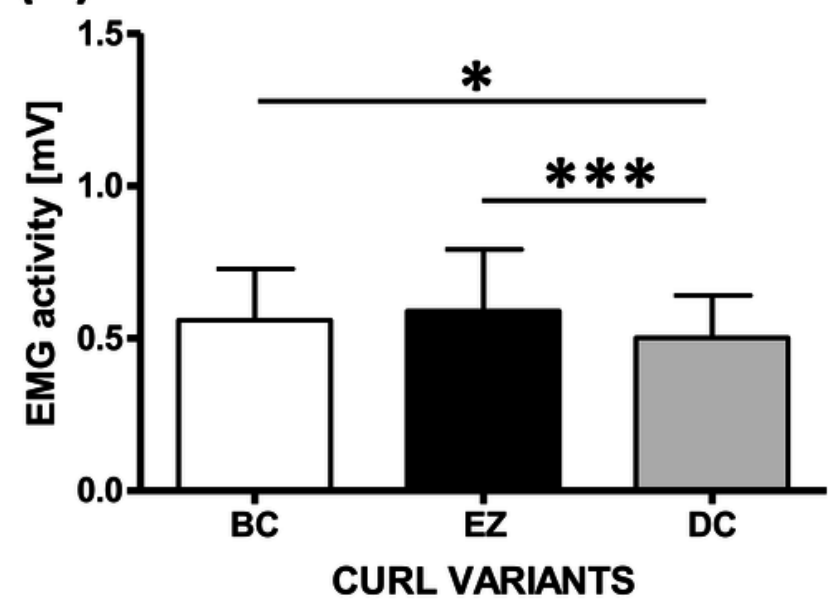

(F) brachioradialis

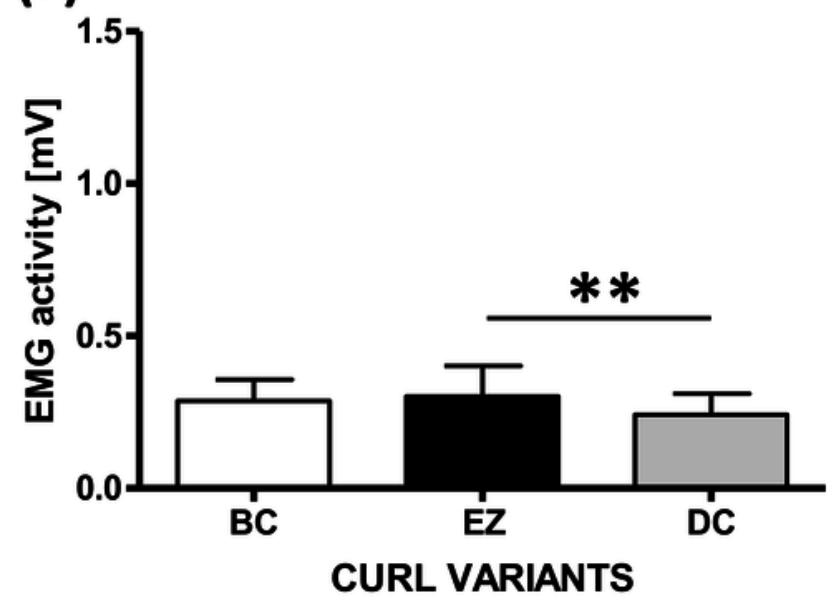

